\title{
The Impact of Play Practice on Chinese Physical Education Pre-Service Teachers Badminton Content Knowledge
}

\author{
Hairui Liu', Wei Wang'2, Yaohui He ${ }^{3}$, Peter Hastie ${ }^{1,2^{*}}$ \\ ${ }^{1}$ School of Kinesiology, Auburn University, USA \\ ${ }^{2}$ College of Physical Education, Hubei Normal University, China \\ ${ }^{3}$ College of Physical Education and Health, East China Normal University
}

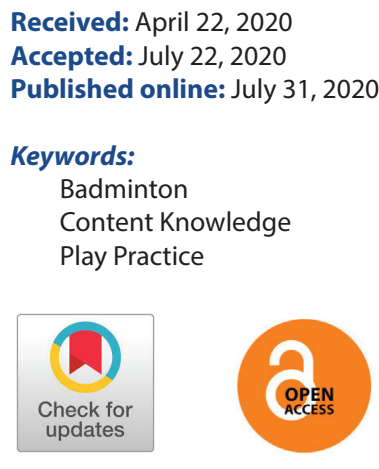

\section{ABSTRACT}

OBJECTIVES This study examined the development of Chinese pre-service physical education teachers' technical skill, tactical understanding, game performance (common content knowledge - CCK), and specialized content knowledge (SCK) during a badminton course incorporating Play Practice instruction.

METHODS Participants were 36 pre-service teachers (31 males, 5 females: age $21 \pm 1.0$ ) majoring in physical education at a university in central China. The students completed a 24 lesson course after a 16 weeks semester. A typical lesson (90 minutes) included a 10-minute warm-up, followed by instruction in two or three technical skills or tactics for 50-55 minutes. The final 25-minutes included the Play Practice aspect of the lesson - a specific game-based challenge to reinforce a technical or tactical aspect of badminton play. The challenge included the three typical pedagogies of Play Practice: focusing, shaping, and enhancing. The French clear test, tactical understanding in badminton, Game Performance Assessment Instrument (GPAl), and student-generated content maps were used to respectively monitor changes in technical skills, tactical understanding, game performance, and specialized content knowledge before and after the course. Parametric statistics were used to compare student outcomes.

RESULTS Statistically significant differences were found on all measures from pre- to post-test, with all showing large effect sizes. In particular, over $75 \%$ of students achieved the benchmark depth of SCK following the course.

CONCLUSIONS The inclusion of Play Practice within a sport instruction course can contribute to various elements that are needed to promote the CCK and SCK of pre-service physical education students.

(c) The Asian Society of Kinesiology and the Korean Academy of Kinesiology

\section{Introduction}

A teacher's content knowledge (the facts, concepts, theories, and principles that are taught and learned in specific academic courses) is considered to be essential for quality instruction [1-3], particularly as it informs pedagogical content

*Correspondence: Peter Hastie, School of Kinesiology, Auburn University, 301 Wire Road, Auburn, AL, USA; Tel: +1 334-750-9189; Fax: +1 334-844-1467; E-mail: hastipe@ auburn.edu knowledge (PCK) [4]. Indeed, Ingersoll et al. [5] reported that teachers without well prepared content knowledge include minimal skill refinements in their instruction, struggle to identify or correct students' performances, and a lack of understanding relative to the purpose of the lesson.

Since its first introduction to the educational literature, content knowledge has been differentiated to include common content knowledge (CCK) and specialized content knowledge 
(SCK) $[1,4]$. CCK is knowledge of the rules and etiquette, and knowledge of techniques and tactics of a specific content area (e.g. knowing how to play a clear in badminton). SCK is knowledge of the sub-domains of instructional tasks (e.g., the task sequence for teaching the badminton clear), representations (e.g., descriptions of the tasks) and errors that are likely with the content (e.g., using the arm to hit the shuttlecock rather than a pullback swing) in teaching CCK.

Previous research in physical education [4] has suggested that CCK can be acquired by simply playing the sport, while SCK must be taught specifically due to its unique in pedagogy. Tsuda's [6] study which examined the relationship between CCK, SCK and performance supported Ward's assertion. In that study, both non-PE majors and PE majors increased their CCK of various sports (volleyball, basketball, badminton, and tennis). However, only PE majors showed significant improvement in SCK given they were the only cohort who received specific SCK training. The evidence from Tsuda [6] implied that a well-organized PETE program can provide sufficient SCK training for pre-service teachers. Despite this important finding, it is important to note that CK instruction accounts for approximately only $10 \%$ of the total credit hours in many PETE programs across the world [7-9]. Further, CCK occupied a larger portion than SCK [7-8] in the programs studied, resulting in potential deficiencies in pre-service teachers' SCK during their professional training.

The teaching of PE in Chinese universities, like many programs across the globe, has an emphasis on learning discrete skills and technical mastery [10-11]. In China, preservice teachers are required to participate in a variety of sports during their first and second years of study, including track and field, basketball, football, volleyball, badminton, dance, and martial arts. Following the second year, students select one major and one minor sport in which to specialize during their third and fourth years of study [12]. In these latter years, the instructional focus follows a direct instructional style in which the focus is on the development of in-depth CCK via knowing and performing sports [12-13]. Tactical content is typically neglected during this direct instruction model [12-13]. As noted, this disconnect between the technical and tactical components will neither help the pre-service teachers to build an authentic capability in game performance nor to develop their content knowledge [14]. Indeed, Ward's [14] study of 384 Chinese secondary in-service teachers demonstrated low levels of SCK in soccer. The study also found that a teacher's teaching experience was negatively associated with their SCK scores.

While a number of game-centered approaches have been developed to address the issue of the separation of technics and tactics in physical education (e.g. Teaching Games for Understanding, Game Sense and Play Practice), to date there have been no efforts to examine their efficacy in promoting students' SCK. By consequence, the purpose of this study was to examine Chinese pre-service physical education teachers' badminton content knowledge following a semester-long course that incorporated Play Practice instruction.

Play Practice was first proposed by Launder [15], with the specific aim of creating skilled and motivated games players. In Launder's description, two key elements make up skillful play. One is "game sense," which includes the ability to use an understanding of the rules, tactics, strategy, and problem solving during the sport play. The second element is having the required technical ability that corresponds with the fundamental skills of a sport. To achieve the aims in Play Practice, three pedagogies are incorporated; focusing, shaping, and enhancing play. Shaping involves modifying practice variables to match the demanding elements of skillful play (e.g. 2 vs. 1 play, size of the court). Focusing play emphasizes instructional specific aspects of game play (play vary angles to open space). Enhancing includes decreasing or increasing the difficulty of the play to promote motivation (adapted racket, balls, or rules in tennis).

The three pedagogies of Play Practice share similarities with Rink's [16] initial content development categories, namely informing (i.e., the initial task), refining (e.g., improving quality of performance), extending (e.g., increasing/decreasing the complexity), and applying (i.e., assessment or games) tasks. Ward et al. [17] expanded these original categories of extending and refining tasks by noting where these occurred in a game context. These tasks were labeled extending-applying tasks (e.g., making the defense active in a 3 vs. 1 game) and refining-applying tasks (e.g., putting specific targets for passing accuracy in a 4 vs. 4 game). Ward et al. [17] also distinguished 
between the features in Rink's [16] original applying tasks by applying nongame tasks that refer to assessing students' performance and applying game tasks where the content of the lesson is used without any particular focus. By consequence, seven content categories can now be used to describe a preservice teachers' use of instructional tasks: informing $(\mathrm{I})$, extending (E), refining (R), extending-applying (EA), refining-applying (RA), applying task-game (AG), and applying nongame (AN) tasks. All of the task categories have been validated in terms of content and concurrent validity by Ward et al. [17].

\section{Methods}

\section{Participants}

The participants in this study were 36 pre-service teachers (31 males, 5 females: age $21 \pm 1.0$ ) who were majoring in physical education at a university in central China. This study was approved by the Institutional Review Board for Research Involving Human Subjects at Hubei Normal University, China (2018001), and all participants provided informed consent.

\section{Instruction}

The badminton course consisted of 24, 90-minute lessons over 16 weeks. Each class began with a 10-minute warm-up, followed by instruction in two or three technical skills or tactics for 50-55 minutes. The final 25-minutes included the Play Practice aspect of the lesson. In the first five weeks of the semester, the teacher selected a specific game-based challenge to reinforce a technical or tactical aspect of badminton play. The challenge included the three typical pedagogies of Play Practice; focusing, shaping, and enhancing. In the remaining weeks of the course, the teacher selected a small group of three or four students to plan, prepare, and introduce a specific challenge. After completing the challenge the remaining students provided feedback concerning the extent to which the challenge was pedagogically sound in developing badminton skill and recommended potential modifications.

\section{Measures}

Three measures were used to assess CCK, while SCK was measured through the creation of content maps by students.
Skill execution. The French Clear Test [18] was used to assess students' skill execution in badminton. The process of French clear begins with the participant receiving a serve and then attempting to hit the shuttle back from across the net to the deepest part of the court. The court is divided into zones, with scores ranging from 0 to 5 depending upon where the shuttlecock lands. Each assessment consists of 10 trials. The French clear test has approved reliable in previous research and replicates a critical skill strongly associated with success in actual badminton play [19].

Tactical understanding in badminton. The video-based game understanding test procedure [20] was used to determine changes in the students' ability to analyze tactical problems by selecting solutions and arguments for their decisions. In this assessment, students watch a series of 19 badminton rallies and then determine the most appropriate response by one player as he or she is getting ready to play a stroke. Students were asked to select the appropriate action out of three alternatives (selected stroke options, SSO) and chose two arguments from a set of ten as to why they chose that option (selected argument options, SAO). Two points are given for selecting the best stroke option, one point for the second best, and no points for the third. Arguments were divided into three groups: 2 points from the best group, one from the second best, and no point for selections in the incorrect group. This test was administered to all participants prior to the first day of instruction and one day after the final lesson.

Game performance. Performance during badminton game play was assessed using the Game Performance Assessment Instrument (GPAI) [21]. In this paper, the elements of decision-making and skill exertion were used. The decisionmaking index (DMI) was scored as the number of appropriate decisions made divided by the number of appropriate and inappropriate decisions made. An appropriate decision was defined as a shot that made the opponent move forwards, backwards or sideways (that is, to take them away from their home or recover position), or one that gave the opponent the shortest time to react to the oncoming shuttlecock (e.g. a smash). The GPAI identified the skill execution index (SEI) as the number of successful skill executions divided by the number of successful and unsuccessful skill executions. A 
successful skill execution was defined as a shot that crossed the net and would have landed in court. Game performance was calculated using the formula $[\mathrm{DMI}+\mathrm{SEI}] / 2$.

Consistent with the recommendations for reliability with the GPAI, only data for which the percentage of agreement between two coders exceeded $80 \%$ were included in the analysis. This reliability was determined through a two-step process. First, four games were randomly selected from the pre- and post-test, which included samples of eight badminton games and 16 participants. From these games, two observers discussed the parameters of both decision and execution components to determine consistency in interpretation. Second, the observers practiced collaborative and independent coding to a point where they reached a greater than $80 \%$ agreement criterion across all nine games. For this study, the final skill-execution and decision-making consensus coding all reached 95\% agreement between the two coders.

Content map design. Prior to, and on completion of the study, each participant designed a badminton content map suitable for a 20-day instructional unit to secondary physical education [17]. A content map is a graphic organizer that defines the content that is to be taught and illustrates the flow and connectedness of the tasks used to develop skillful performance with beginners and ending with what would be considered more advanced skill [22]. Participants were instructed to list and show the sequence of instructional tasks necessary to teach badminton to secondary school students, and to diagram relationships among the content such as when they might combine skills, and combine skills and tactics.

To analyze the depth of content development presented in the content maps, the protocol used by Ward et al. [17] was followed. First, each instructional task in a map was coded as informing (I), extending (E), extending applying tasks (EA), refining $(\mathrm{R})$, refining applying tasks (RA), applying task game $(\mathrm{AG})$, or applying task nongame (AN). Second, the formula $(\mathrm{E}+\mathrm{EA}+\mathrm{R}+\mathrm{RA}+\mathrm{AG}+\mathrm{AN}) / \mathrm{I}$ was used to calculate a ratio to reflect the depth of content knowledge. This formula is based on an assumption that an informing task is an initial task in a sequence against which all other tasks can be compared because it represents the starting place for the teaching of the content. The formula creates an index score that represents the number of tasks that are developed beyond the informing task. Thus, an index score of 2.0 would mean that there were two other tasks (e.g., extending and applying) that were used to develop that skill. Research has shown that an index score of 3.0 could be considered as a depth of SCK benchmark [23].

\section{Data Analysis}

Pearson-product correlations were conducted to examine the relationships among the four dependent variables: French clear, tactical understanding, game performance, and content maps. No outliers have been identified in four measures (Q-Q plots and scatter plots). In addition, normality (KolmogorovSmirnov test) and homogeneity (Levene's test) are also checked before further analysis. The results showed that normality and equal variance are not violated in all four tests. A paired sample t-test was used to analyze the difference between the pre- and post-measures for all measures, with the significance level set at 0.013. Effect sizes (Cohen's d) were used to determine the meaningful difference among variables of interest. Effect sizes of $<0.2,0.2-0.5,0.5-0.8$, and $>0.8$ represented extremely low, low, medium, and high effect sizes respectively [24].

\section{Results}

The scores in French clear, tactical understanding, GPAI, and content maps are presented in <Table 1>. Results of the t-tests showed there were statistically significant improvements in all four measures from pre-to post-test after the Play Practice badminton course. All comparisons showed large effect sizes. < Figure $1>$ presents the extent to which students were greater or less than Ward's cut-off for the benchmark depth of SCK. It can be seen in this figure that over $75 \%$ of students achieved a score of 3 or greater following the course. This score of 3 is considered the benchmark depth of SCK.

\section{Discussion}

The purpose of this study was to determine whether the inclusion of Play Practice instruction would positively impact Chinese pre-service teachers' content knowledge of badminton. The results showed that the three pedagogies of shaping, focusing, and enhancing play seemed particularly 
Table 1. Technique, Tactics, GPAI and SCK scores

\begin{tabular}{llllll}
\hline Variables & $\begin{array}{l}\text { Pre-test } \\
(\boldsymbol{M}, \boldsymbol{S D})\end{array}$ & $\begin{array}{l}\text { Post-test } \\
(\boldsymbol{M}, \boldsymbol{S D})\end{array}$ & $\boldsymbol{t}$ & $\mathrm{p}$ & $\mathrm{d}$ \\
\hline French Clear & $29.58(7.71)$ & $46.47(1.87)$ & 13.99 & $<.001$ & 2.33 \\
$\begin{array}{l}\text { Tactical } \\
\text { understanding }\end{array}$ & $53.28(16.15)$ & $88.75(8.68)$ & 12.06 & $<.001$ & 2.01 \\
GPAI & $2.82(1.19)$ & $6.15(2.00$ & 11.37 & $<.001$ & 1.89 \\
SCK & $1.26(0.90)$ & $3.69(1.24)$ & 10.33 & $<.001$ & 1.72 \\
\hline
\end{tabular}

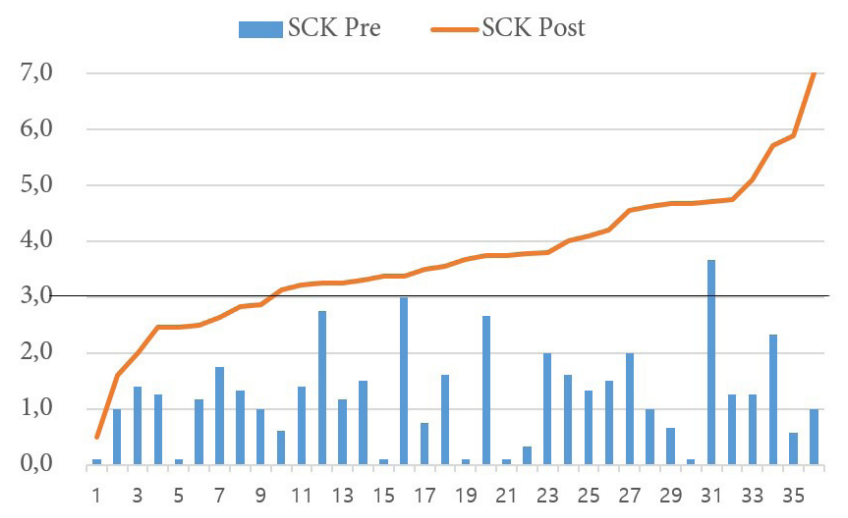

Figure 1. Comparisons of SCK scores prior to and on completion of the course.

effective in achieving this goal.

In terms of CCK, the primary pedagogy of Play Practice begins with an analysis of the activity and then determining which aspects of skilled play are most important for the particular group of learners [15]. In this way, one would expect that those elements of CCK (knowing how to perform techniques and tactics) would be promoted by participation in challenges designed specifically to address current deficiencies in performance. This theory is indeed supported by the results of this study.

Most notably from the data in this study is that the SCK pre-test scores of students were particularly low, reinforcing previous results indicating deficiencies in Chinese teachers' SCK [14]. These low scores also reinforce the idea that SCK is not knowledge that a nonteaching or non-coaching person would possess, and because of this needs to be explicitly taught. However, post-test data show significant improvements so that the majority of students ( $>75 \%)$ achieved a depth of SCK considered to be an adequate benchmark $[17,23]$. It is hypothesized here that that greatest contribution to the development of SCK came from the challenges students were asked to design during the course. Recall that the final 25 minutes of lessons involved students in progressively designing, teaching and playing technical and tactical related challenges to stimulate "game sense" skill and skill. In particular, the students were required to design tasks that would first, address a skill weakness that perceived as occurring in the class (a component of SCK) and then using shaping, focusing, and enhancing tasks to improve these weaknesses.

Previous research $[6-9,17,22-23]$ has shown that lowerlevel content maps are dominant by informing tasks that neglect other tasks, such as extension, applying, and refiningtasks. To create a competent content map, the basic principle is to connect information with extension, applying, refiningtasks rather than merely constrained in informing tasks [2223]. Given previous research suggesting that SCK needs to be explicitly taught in the Physical Education Teacher Education program rather than acquired in engagement in field teaching or experiences [5-7,25], we suggest that the participation in a course where students are first exposed to these connections, and later, are required to design then, is one strategy that teacher preparation programs could use to improve SCK in sports-based courses.

\section{Limitations of the Study}

Like all studies, this study has limitations. First, this study is a pre-post design, and as such, some students may have improved simply due to the familiarity of the testing protocols. Second, the lack of a control group is another limitation that may prevent us from interpreting the results precisely in this study. Future research highly suggests recruiting more participants to validate Play Practice's effects in content knowledge development with a quasi- or full experimental design.

\section{Conclusions}

A Play Practice intervention can improve all aspects of preservice teachers' badminton competency, including skills and game performance, tactical understanding, and specialized 
content knowledge (SCK). We suggest PETE programs consider applying Play Practice to their formal training of pre-service teachers' content knowledge development.

\section{References}

1. Ball DL, Thames MH, Phelps G. Content knowledge for teaching: What makes it special? J Teach Educ. 2008; 59(5):389-407.

2. Shulman LS. Knowledge and teaching: Foundations of the new reform. Harvard Educ Rev. 1987; 57(1):1-22.

3. Siedentop D. Content knowledge for physical education. J Teach Phys Educ. 2002; 21(4):368-377.

4. Ward P. Content matters: Knowledge that alters teaching. In: Housner L, Metzler M, Schempp P, editors. Historic traditions and future directions of research on teaching and teacher education in physical education. Morgantown WV: Fitness Information Technology. 2009, p 345-356.

5. Ingersoll C, Jenkins JM, Lux K. Knowledge development in early field teaching experiences. J Teach Phys Educ. 2014; 33:363-382.

6. Tsuda E, Ward P, Li Y, Higginson K, Cho K, He Y, Su J. Content knowledge acquisition in physical education: Evidence from knowing and performing by majors and nonmajors. J Teach Phys Educ. 2019; 38:221-232.

7. Kim I, Lee YS, Ward P, Li W. A critical examination of content knowledge courses in physical education teacher education programs. J Teach Phys Educ. 2015; 34:59-75.

8. Ward P. Re-conceptualizing pedagogical content knowledge. Res Q Exercise Sport Supp. 2012; 83:A3.

9. Ward P, Ince LM, Iserbyt P, Kim I, Lee, YS, Li W, Lui W. International physical education teacher education physical activity content knowledge study. Res Q Exercise Sport Supp. 2013; 84:A4.

10. Guoyong D, Wang J, Zhai M, Wang T, Lu C. The primary and secondary Chinese physical education teachers' professional development. J Phys Educ. 2015; 4:76-82.

11. Zuo X, Zhou M. The constraints in technical driven physical education pre-service teacher training - a reflection in college PETE program. Sports Science. 2005; 4:75-77.

12. Wang L. Teaching perspectives of preservice physical education teachers: The Shanghai experience. Phys Educ Sport Peda. 2014; 19:451-465.

13. Zhao Q, Li W. Zhou W. The Current status and future reforming directions of Chinese undergraduate physical education teacher education training program and curriculum in context of national standards for teaching quality for undergraduate sport majors. J Nanjing Sport Inst (Soc Sci). 2017; 5:115-119.

14. Ward P, He Y, Wang XW. Chinese secondary physical education teacher's depth of specialized content knowledge in soccer. J Teach Phys Educ. 2018; 37:101112.

15. Launder A. Play practice: The games approach to teaching and coaching sports. Champaign, IL: Human Kinetics. 2001. 187p.

16. Rink JE. Development of a system for the observation of content development in physical education [dissertation]. [Columbus ( $\mathrm{OH})$ ]: The Ohio State University; 1979. 368p.

17. Ward P, Dervent F, Lee YS, Ko B. Kim I. Tao W. Using content maps to measure content development in physical education: Validation and application. J Teach Phys Educ. 2017; 36:20-31.

18. Scott MG, Carpenter A, French A, Kuhl L. Achievement examinations in badminton. Res Q Exercise Sport. 1941; 12:242-253.

19. Rink JE, French KE, Graham KC. Implication for practice and research. J Teach Phys Educ. 1996; 15:490-502.

20. Blomqvist MT, Luhtanen P, Laakso L, Keskinen E. Validation of a video-based game-understanding test procedure in badminton. J Teach Phys Educ. 2000; 19:325-337.

21. Oslin J L, Mitchell SA, Griffin LL. The Game Performance Assessment Instrument (GPAI): Development and preliminary validation. J Teach Phys Educ. 1998; 17:231-243.

22. Ward P, Lehwald C, Lee YS. Content maps: A teaching and assessment tool for content knowledge. J Phys Educ Rec 
Dance. 2015; 86(5):46-54.

23. Dervent F, Tsuda E, Devrilmez E, Ward P. Content development coding assessment manual. Version 2.1. 2016: https://u.osu.edu/ltpe/.

24. Faul F, Erdfelder E, Lang AG, Buchner A. G* Power 3: A flexible statistical power analysis program for the social, behavioral, and biomedical sciences. Behavior Research Methods. 2007; 39:175-191.

25. Ward P, Tsuda E, Dervent F, Devrilmez E. Differences in the content knowledge of those taught to teach and those taught to play. J Teach Phys Educ. 2018; 37:59-68. 